\begin{tabular}{|c|l|}
\hline Title & Mechanism of photoinduced anisotropy in chal cogenide glasses \\
\hline Author(s) & Tanaka, K.; Ishida, K.; Y oshida, N. \\
\hline Citation & $\begin{array}{l}\text { Physical Review B, 54(13), 9190-9195 } \\
\text { https://doi.org/10.1103/PhysRevB.54.9190 }\end{array}$ \\
\hline Issue Date & 1996-10-01 \\
\hline Doc URL & http://hdl.handle.net/2115/5731 \\
\hline Rights & Copyright $\odot$ 1996 A merican Institute of Physics \\
\hline Type & article \\
\hline File Information & PRB54-13.pdf \\
\hline
\end{tabular}

Instructions for use 


\title{
Mechanism of photoinduced anisotropy in chalcogenide glasses
}

\author{
K. Tanaka, K. Ishida, and N. Yoshida \\ Department of Applied Physics, Faculty of Engineering, Hokkaido University, Sapporo 060, Japan
}

(Received 8 May 1996; revised manuscript received 13 June 1996)

\begin{abstract}
Structural and optical anisotropies have been investigated in some chalcogenide glasses exposed to illumination of linearly polarized light. X-ray diffraction patterns of illuminated $\mathrm{As}_{2} \mathrm{~S}_{3}$ manifest a structural modification at $\sim 1 \AA^{-1}$. Photoinduced birefringence in $\mathrm{As}_{2} \mathrm{~S}_{3}$ becomes maximal at some temperature, which is higher for illumination with a lower photon energy. The photoinduced birefringence becomes greater in the order of $\mathrm{As}_{2} \mathrm{Se}_{3}, \mathrm{As}_{2} \mathrm{~S}_{3}$, and $\mathrm{Se}$, which is the same with the order of the natural birefringence in the corresponding crystals. These observations suggest that the photoinduced anisotropy arises from orientation of quasicrystalline clusters. The model is compared with photoinduced anisotropies observed in other materials. [S0163-1829(96)06138-3]
\end{abstract}

\section{INTRODUCTION}

Chalcogenide glasses are known to exhibit a variety of photoinduced phenomena, ${ }^{1-4}$ among which the photoinduced anisotropy ${ }^{5,6}$ has aroused considerable interests in fundamental $^{7-20}$ and applied researches. ${ }^{21,22}$ The phenomenon refers to the observations that chalcogenide glasses exhibit optical anisotropies, i.e., birefringence and dichroism, when exposed to linearly polarized light. The photoinduced anisotropy is metastable after cessation of illumination, while it can be erased with illumination of circularly or unpolarized light or with annealing. In addition, the anisotropic principal axis can be altered by changing the direction of the electric field vector of polarized light. That is, we can induce, erase and change the optical anisotropy, repeatedly. It is also demonstrated very recently that even circularly or unpolarized light can induce the anisotropy, if light is incident upon a sample from sideward directions. ${ }^{16,17,19}$

The mechanism of the photoinduced anisotropy can be understood in a phenomenological sense through Fritzsche's model. ${ }^{16} \mathrm{He}$ has proposed that isotropic dielectric tensors characterizing annealed glasses become anisotropic with illumination, since structural elements having the tensor components parallel to the electric field of illumination are excited preferentially, which may be rotated to other directions when relaxed. Therefore, consistent with experimental observations, ${ }^{10,19}$ the negative anisotropy is induced; that is, the birefringence $\Delta n=n(\|)-n(\perp)$ and the dichroism $\Delta \alpha$ $=\alpha(\|)-\alpha(\perp)$ are negative, where $n$ is the refractive index at transparent wavelengths and $\alpha$ is the absorption coefficient at around optical absorption edges, and the symbols $\|$ and $\perp$ refer to the electric field vectors of probe light parallel and perpendicular to that of excitation light.

In contrast, microscopic atomic structures giving rise to the anisotropy are largely speculative. Quenched-in strains, which may be generated by illumination, seem to be less probable. ${ }^{23}$ Alternatively, we may assume a variety of atomic orientations of different length scales such as directional changes in lone-pair electron orbitals, ${ }^{7,11,13}$ covalent bonds, ${ }^{13}$ dangling bonds, ${ }^{18,20}$ and molecular units. ${ }^{9,12}$ In a model, which may be termed as the crystalline model, structural elements similar to, but not necessarily the same with, the corresponding crystalline structure are assumed to be oriented with illumination. ${ }^{5,6,8,19}$

In order to elucidate the microscopic mechanism of the photoinduced anisotropy, the structural element responsible for the optical anisotropy should be revealed. The present experimental studies including $\mathrm{x}$-ray and birefringence measurements for elemental and stoichiometric chalcogenide glasses strongly suggest that the crystalline model is more plausible. In addition, it is shown that localized photoexcited carriers can effectively produce the optical anisotropy.

\section{EXPERIMENTS}

$\mathrm{X}$-ray and birefringence measurements were performed using annealed films of $\mathrm{As}_{2} \mathrm{~S}_{3}, \mathrm{As}_{2} \mathrm{Se}_{3}$ and Se. These films were prepared by evaporation, which were then annealed in a flowing $\mathrm{Ar}$ atmosphere at the glass-transition temperatures for $1 \mathrm{~h}$. In details, for the $\mathrm{X}$-ray measurements, $\mathrm{As}_{2} \mathrm{~S}_{3}$ films of about 4-8 $\mu \mathrm{m}$ thickness deposited onto mica films, Al foils, and $\mathrm{Si}$-crystal wafers were examined. On the other hand, for the optical measurements, $\mathrm{As}_{2} \mathrm{~S}_{3}$ films with thickness of $1-50 \mu \mathrm{m}, \mathrm{As}_{2} \mathrm{Se}_{3}$ films of $2 \mu \mathrm{m}$, and Se films of $1-15 \mu \mathrm{m}$ deposited on to oxide-glass substrates were employed. For accurate optical measurements, thick films peeled off from the substrates were preferred, since the substrates exhibited inhomogeneous birefringence. Subsidiary measurements for bulk As-S glasses have also been made.

X-ray diffraction was investigated for the reflection (Bragg) and the transmission (Laue) arrangement. To obtain accurate results, the substrate must be rigid in the reflection arrangement, and accordingly the samples deposited upon $\mathrm{Si}$ wafers were inspected. On the other hand, in the transmission arrangement, the substrate should be transparent to $\mathrm{x}$ rays, so that $\mathrm{As}_{2} \mathrm{~S}_{3}$ films deposited upon $\mathrm{Al}$ foils and mica plates were employed. X-ray diffraction patterns were monitored at room temperature using a Rigaku RU-300 system operating with a $\mathrm{Cu}$ rotating target at $50 \mathrm{kV}$ and $250 \mathrm{~mA}$ and a scintillation counter equipped with a graphite monochromator. An annealed $\mathrm{As}_{2} \mathrm{~S}_{3}$ sample was mounted on a goniometer, and exposed to light illumination in situ, which was necessary to detect subtle modifications induced by illumination. Light emitted from a $250 \mathrm{~W}$ ultrahigh-pressure $\mathrm{Hg}$ lamp 


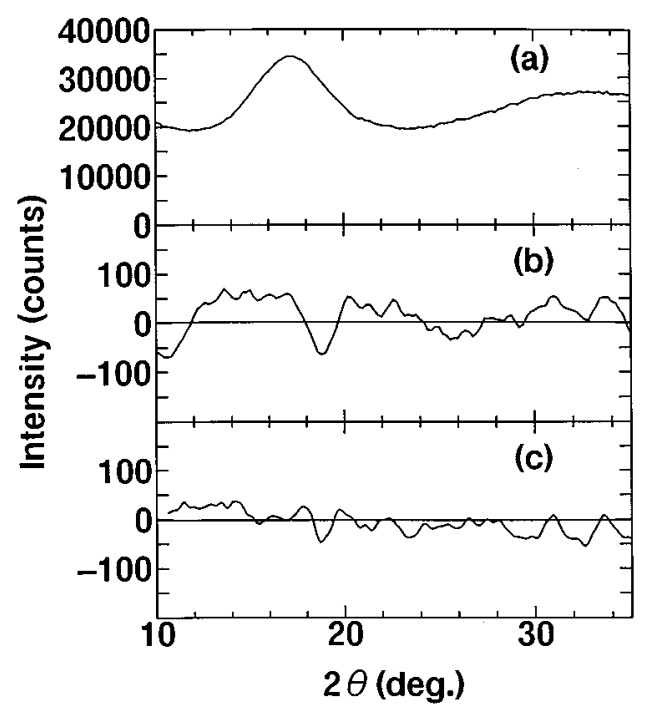

FIG. 1. An x-ray diffraction pattern of an annealed $\mathrm{As}_{2} \mathrm{~S}_{3}$ film (a), and the differences $I_{x}-I_{y}$ measured in the transmission (b) and the reflection (c) arrangement. $I_{x}$ and $I_{y}$ are defined as the x-ray intensities diffracted from a sample which has been illuminated with $x$ - and $y$-polarized light. (a) and (b) are obtained by averaging and smoothing a few data.

was passed through a polarization sheet, and it was incident normally upon the film surface. The polarization was varied at $x$ (horizontal) and $y$ (vertical) directions. The exposure time was $\sim 1 \mathrm{~h}$, which was fixed by taking the time dependence of the optical birefringence into account.

The birefringence $\Delta n$ was measured using a transmissiontype ellipsometer. ${ }^{19}$ Probe light was provided from a He-Ne laser, the photon energy and the intensity being $2.0 \mathrm{eV}$ and $\sim 1 \mathrm{~mW} / \mathrm{cm}^{2}$. In this instrument, the phase difference between the two orthogonally-polarized light transmitted through a sample could be measured. The result was used to calculate the birefringence, after taking multiple-interference effects into account. ${ }^{24}$ The birefringence was induced with monochromatic light emitted from He-Ne lasers, ultrahighpressure Hg lamps, and a Xe lamp, in conjunction with some optical filters. The light intensity ranged over $10^{-3}-10^{3}$ $\mathrm{W} / \mathrm{cm}^{2}$. A sample was mounted in an optical cryostat, and the temperature was varied at $80-430 \mathrm{~K}$. For simplicity, light excitation and birefringence measurements were made at the same temperatures, while it was confirmed previously that the temperature dependence did not change if probing was made at temperatures equal to or lower than that of light excitation. ${ }^{19}$ Annealing characteristics of the photoinduced birefringence was also investigated, in which illuminated samples were annealed in an Ar atmosphere for $0.5 \mathrm{~h}$.

\section{RESULTS}

\section{A. X-ray diffraction}

Figure 1 shows typical $\mathrm{x}$-ray results for $\mathrm{As}_{2} \mathrm{~S}_{3}$. Figure 1(a) is a diffraction pattern of an annealed film. The result is obtained for the transmission arrangement, while similar profiles have been recorded for the reflection arrangement. We see the so-called first sharp diffraction peak (FSDP) at $2 \theta$

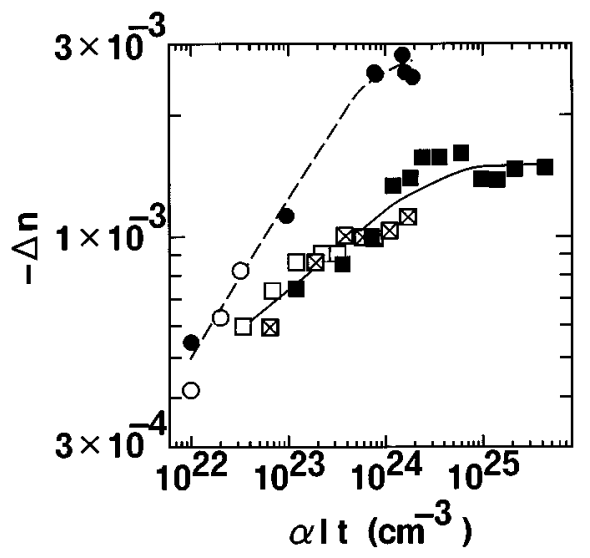

FIG. 2. The photoinduced birefringence $\Delta n$ for $2.0 \mathrm{eV}$ (solid line) and $2.3 \mathrm{eV}$ (dashed line) excitation as a function of $\alpha I t$, where $\alpha$ is the absorption coefficient, $I$ the incident light intensity, and $t$ the exposure time. $I$ is changed at $5(\square), 50(\nabla)$, and $1000(\square)$ $\mathrm{W} / \mathrm{cm}^{2}$ for $2.0 \mathrm{eV}$ light and $2 \mathrm{~mW} / \mathrm{cm}^{2}(\bigcirc)$ and $1 \mathrm{~W} / \mathrm{cm}^{2}(\bigcirc)$ for $2.3 \mathrm{eV}$ light.

$\simeq 17^{\circ}$, i.e., the wave number of $\sim 1 \AA^{-1}$, and the second halo peak at $\sim 32^{\circ}$, which are consistent with the previous results. ${ }^{1,3,25}$

Figures 1(b) and 1(c) show the differences $I_{x}-I_{y}$ for the transmission and the reflection arrangement, where $I_{x}$ and $I_{y}$ are the diffracted $\mathrm{x}$-ray intensity after illumination with $x$ and $y$-polarized light. Since the photoinduced structural modification was assumed to be subtle, $\mathrm{x}$-ray measurements have been repeated for several samples. Actually, the intensity difference was noisy, and the difference appeared to be only the order of $10^{-3}$ of the diffracted intensity. Hence, some data were averaged and smoothed. As shown in the figures, the intensity differences appear to be still noisy, while two reproducible features can be pointed out; for the transmission arrangement (b) $I_{x}-I_{y}>0$ at $2 \theta \simeq 12^{\circ}-22^{\circ}$, except at $\sim 19^{\circ}$, and for the reflection arrangement (c) $I_{x}-I_{y} \simeq 0$ for all the angles investigated. Quantitatively, the difference observed in (b) has been smaller by about an order of magnitude than that induced with the reversible photostructural change, ${ }^{1,4,25}$ which may be consistent with the refractive-index changes; $\sim 0.03$ for the reversible change ${ }^{2}$ and $\sim 0.002[=-n(\|)+n(\perp)]$ for the photoinduced birefringence (described below). The response appearing at $\sim 19^{\circ}$ both in (b) and (c) may be due to a trace of the reversible change.

The noisy signal shown in (b) makes quantitative evaluation difficult. However, the position and the width of the $\mathrm{x}$-ray change seem to imply that some structural changes with a periodicity of $\sim 0.5 \mathrm{~nm}$ and an extension of $\sim 1 \mathrm{~nm}$ are induced with illumination of linearly-polarized light. ${ }^{26}$

\section{B. Birefringence}

Figure 2 shows the photoinduced birefringence $\Delta n$ in $\mathrm{As}_{2} \mathrm{~S}_{3}$ as a function of $\alpha \mathrm{It}$, which approximates the absorbed photon dose, for 2.0 and $2.3 \mathrm{eV}$ excitation. Here, $\alpha$ is the absorption coefficient, $I$ the incident light intensity, and $t$ the exposure time. We see that $\Delta n \simeq-0.002,{ }^{27}$ which is comparable to the previous result obtained using a wave-guiding measurement. ${ }^{2}$ In detail, $2.0 \mathrm{eV}$ excitation gives a smaller 


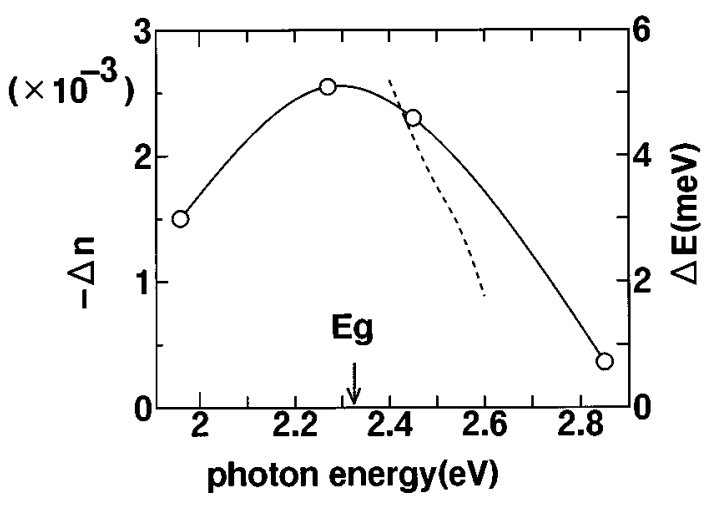

FIG. 3. The photoinduced birefringence $\Delta n$ in $\mathrm{As}_{2} \mathrm{~S}_{3}$ at room temperature as a function of excitation photon energy. Also shown by a dotted line is the photoinduced dichroism (the difference of the absorption-edge shift) $\Delta E$ (Ref. 10), which is read by the righthand-side scale. The Tauc optical bandgap energy $E_{g}$ is indicated.

birefringence than that induced by $2.3 \mathrm{eV}$ excitation. In addition, we see that the reciprocity law between the light intensity and the exposure time is satisfied; for instance, for 2.0 $\mathrm{eV}$ illumination, the light intensity is varied at three-orders of magnitude, while $\Delta n$ seems to be governed only by the absorbed photon density. We may conclude that the photoinduced birefringence manifests no light-intensity dependence, which is consistent with the observations obtained for the photoinduced dichroism. ${ }^{10}$

Figure 3 shows the photoinduced birefringence in $\mathrm{As}_{2} \mathrm{~S}_{3}$ as a function of excitation photon energy. Here, illumination is continued until saturation ( $1 \mathrm{~h}-1$ day) at room temperature. We see that $-\Delta n$ is maximal at $2.3 \mathrm{eV}$, and it decreases at lower and higher energies. This photon-energy dependence is also consistent with that of the photoinduced dichroism $\Delta E$, which shows a decrease at $2.4-2.6 \mathrm{eV}$ with an increase in the photon energy. ${ }^{5,10}$ Here, $\Delta E$ is defined as $E(\|)-E(\perp)$, where $E$ denotes the photon energy at $\alpha \simeq 5 \times 10^{3} \mathrm{~cm}^{-1},{ }^{10}$ and accordingly $\Delta E>0$ means the negative dichroism, $\Delta \alpha<0$. Note that the Tauc optical bandgap $E_{g}$ in $\mathrm{As}_{2} \mathrm{~S}_{3}$ is $\sim 2.32 \mathrm{eV},{ }^{3}$ and accordingly we may assume that the photoinduced anisotropy is maximal for bandgap illumination of $\hbar \omega \simeq E_{g}$. This photon-energy dependence is in contrast to that of the photodarkening, which is nearly constant for light with $\hbar \omega \geqslant E_{g}$ in $\mathrm{As}_{2} \mathrm{~S}_{3}{ }^{2}$

Interestingly, however, the spectral dependence changes with the temperature at which the sample is illuminated. Figure 4 shows the photoinduced birefringence in $\mathrm{As}_{2} \mathrm{~S}_{3}$ as a function of temperature for excitation of 2.0 and $2.3 \mathrm{eV}$. We see that $-\Delta n$ induced with $2.3 \mathrm{eV}$ is maximal at $300 \mathrm{~K}$, while for $2.0 \mathrm{eV}$ it is maximal at $380 \mathrm{~K}$, the latter being consistent with the previous result. ${ }^{19} \mathrm{We}$ also note that the temperature dependence of $2.3 \mathrm{eV}$ is very similar to that of the photoinduced dichroism induced by $2.4 \mathrm{eV}$ illumination. ${ }^{10}$ This temperature dependence is distinctly different from that of the reversible photodarkening, ${ }^{2,10}$ which only decreases with an increase in temperature without showing no peaks. We can argue therefore, that thermal energy is indispensable for the photoinduced anisotropy.

The annealing-temperature dependence of the birefringence, which is induced at $300 \mathrm{~K}$ by $2.3 \mathrm{eV}$ excitation, is also shown in Fig. 4 by a dashed line. As shown in Fig. 4,

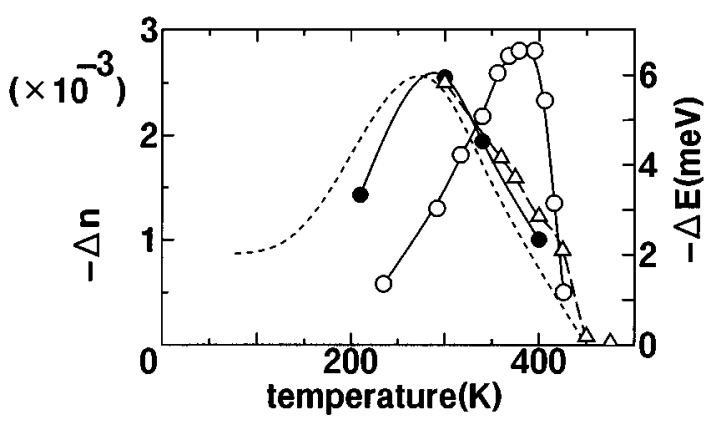

FIG. 4. The photoinduced birefringence $\Delta n$ in $\mathrm{As}_{2} \mathrm{~S}_{3}$ as a function of temperature for $2.0(\bigcirc)$ and $2.3(0) \mathrm{eV}$ excitations. Light excitation and birefringence measurements are made at the same temperatures. For the dashed line with the triangle symbols $(\triangle)$, the horizontal scale indicates the annealing temperature, and illumination and probe are made at room temperature. Also plotted by a dotted line is the photoinduced dichroism $\Delta E$, which is read by the right-hand-side scale (Ref. 10).

the birefringence disappears, with annealing at $450 \mathrm{~K}$, which is just below the glass-transition temperature. ${ }^{3}$ It is often stated that the induced anisotropy can be erased with annealing at temperatures substantially lower than the glasstransition temperature. ${ }^{5,9,15,16}$ The present result demonstrates, however, that, although thermal recovery starts at just above the illumination temperature, complete erasure of the anisotropy requires an annealing at just below the glasstransition temperature, at least, in $\mathrm{As}_{2} \mathrm{~S}_{3}{ }^{28}$ Also in Se, the birefringence can be induced at room temperature (see, Fig. 5), which is just below the glass-transition temperature for $\mathrm{Se}^{3}$

Figure 5 shows the temperature dependence of the photoinduced birefringence in three chalcogenide glasses. The birefringence is induced with bandgap illumination, i.e., $2.0 \mathrm{eV}$ for $\mathrm{Se}$ and $\mathrm{As}_{2} \mathrm{Se}_{3}$, and $2.3 \mathrm{eV}$ for $\mathrm{As}_{2} \mathrm{~S}_{3}$. We see that all the materials exhibit qualitatively similar temperature dependences, in which the most remarkable feature may be the fact that the maximal birefringence $|\Delta n|$ increases with the order of $\mathrm{As}_{2} \mathrm{Se}_{3}, \mathrm{As}_{2} \mathrm{~S}_{3}$, and Se. This material dependence is completely different from that in the reversible photodarkening, in which $\mathrm{As}_{2} \mathrm{~S}_{3}$ exhibits the greatest change among these three materials. $^{2}$

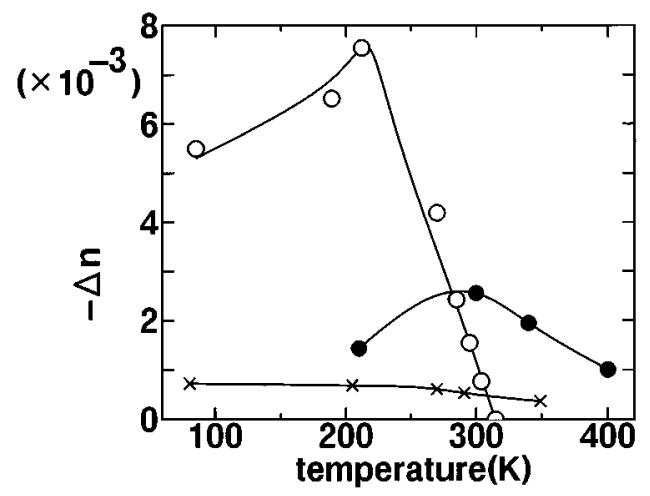

FIG. 5. The photoinduced birefringence $\Delta n$ induced by bandgap illumination in $\mathrm{Se}(\mathrm{O}), \mathrm{As}_{2} \mathrm{~S}_{3}(\mathbf{O})$ and $\mathrm{As}_{2} \mathrm{Se}_{3}(\times)$ as a function of the temperature at which excitation and probe are performed. 


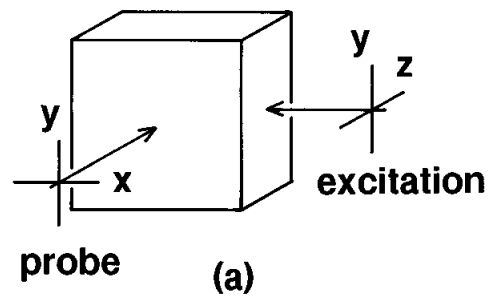

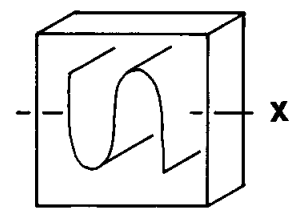

(b)

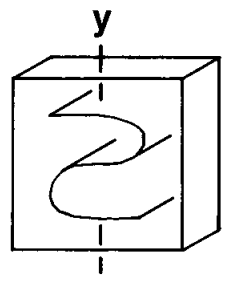

(c)

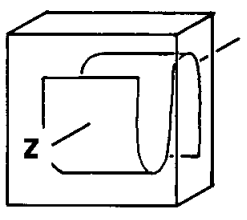

(d)
FIG. 6. Schematic illustrations of transversal illumination (a), and an oriented layer cluster which may be produced with illumination of light linearly polarized along the $x$ (b), $y$ (c) and the $x$ direction (d). Only one oriented cluster is illustrated for simplicity.

\section{DISCUSSION}

In the beginning, it seems to be valuable to summarize the previous study ${ }^{19}$ which gives motivation for the present work. In the study, the photoinduced birefringence in $\mathrm{As}_{2} \mathrm{~S}_{3}$ was examined under transversal excitation [see, Fig. 6(a)], in which a probe beam is propagated along the $z$ direction and it measures the birefringence $\Delta n$ defined as $n(x)-n(y)$. On the other hand, excitation light is propagated along the $x$ direction with the polarization being varied in the $y-z$ plane. Then, it has been found that $\Delta n_{y z} \simeq \Delta n_{y} / 2$, where $\Delta n_{y z}$ is the birefringence induced with circularly polarized (or unpolarized) light and $\Delta n_{y}$ is the birefringence induced with linearly polarized light along the $y$ axis.

This result appears to be inconsistent with an idea predicted from the Fritzsche's model. ${ }^{16}$ According to the model, we may expect that, upon the sideward excitation using circularly polarized (or unpolarized) light, microstructures having the dielectric tensors lying in the $y-z$ plane will be transferred to the $x$-oriented structures. In contrast, the $y$-polarized excitation will induce transformations from the $y$ to the $x$ and $z$ orientations. Accordingly, the proposed model may predict $\Delta n_{y z}>\Delta n_{y}$, which is in disagreement with the observations.

However, we can reconcile the Fritzsche's model with the experimental result, taking a crystalline model into account. ${ }^{19}$ We here note that the chalcogenide crystals of interest possess low-dimensional structures. For instance, crystalline $\mathrm{As}_{2} \mathrm{~S}_{3}$ has a layer-type structure with the in-plane refractive indices of $\sim 3.0$ and the out-of-plane index of $2.5 .^{29,30}$ On the other hand, Fritzsche has proposed that light illumination excites preferentially some elements having higher refractive indices (dielectric tensors), and accordingly after illumination the refractive index along the electric field of light becomes smaller. ${ }^{16}$ Therefore, if we may assume that remnants of crystalline clusters are contained in amorphous networks and/or these are generated with illumination, $y$-polarized illumination can produce the structure such as shown in Fig. 6(c). That is, the layer planes will be normal to
TABLE I. Maximal photoinduced birefringences $\Delta n=n(\|)$ $-n(\perp)$ in glasses and the natural birefrigences $\Delta n_{n}=n(s)-n(l)$ in the corresponding crystalline materials. The photoinduced birefringence is evaluated from Fig. 5. The natural birefringence is evaluated as follows: For Se, $n(s)=2.8$ and $n(l)=3.6$, which are the refractive indices perpendicular and parallel to the chain axis in the hexagonal form (Ref. 43). For $\mathrm{As}_{2} \mathrm{~S}_{3}$, the details are described in the text. For $\mathrm{As}_{2} \mathrm{Se}_{3}$, which is polymorphous to $\mathrm{As}_{2} \mathrm{~S}_{3}$, no experimental result seem to exist, and accordingly the birefringence is estimated from a theoretical result for the dielectric constant (Ref. $44)$.

\begin{tabular}{lcc}
\hline \hline Material & Photoinduced & Natural \\
\hline $\mathrm{Se}$ & -0.007 & -0.8 \\
$\mathrm{As}_{2} \mathrm{~S}_{3}$ & -0.002 & -0.5 \\
$\mathrm{As}_{2} \mathrm{Se}_{3}$ & -0.0008 & -0.3 \\
\hline
\end{tabular}

the $y$ axis, the structure which can give rise to substantial birefringence $\left(\Delta n_{y}>0\right)$. In contrast, the circularly polarized (or unpolarized) light produces the structure in which the layer normal distributes between the $y$ and $z$ directions [Figs. 6(c) and 6(d)]. In this case, the crystalline clusters with the layer normal aligning toward the $z$ direction [Fig. 6(d)] cannot contribute to the birefringence $\left(\Delta n_{z}=0\right)$, while the $y$-directed clusters [Fig. 6(c)] can provide some birefringence $\left(\Delta n_{y}>0\right)$. Therefore, the fact that $\Delta n_{y}>\Delta n_{y z}$ can be understood straightforwardly. Note that chalcogenide glasses exhibit photocrystallization phenomena, ${ }^{2}$ and the process may be similar to the present reaction.

The x-ray result shown in Fig. 1 is consistent with the crystalline model. In the experiment, excitation light polarized along the $x$ and $y$ directions can give rise to the structures illustrated in Figs. 6(b) and 6(c), respectively. Then, the $\mathrm{X}$-ray intensity at around the FSDP position will unchange, $I_{x}-I_{y}=0$, for the reflection arrangement, but it will be $I_{x}-I_{y}>0$ for the transmission arrangement, since the layer structure illustrated in Fig. 6(b) can give rise to a Bragg peak at around the FSDP position for the transmission arrangement. This prediction is in agreement with the experimental result.

The composition dependence of the photoinduced birefringence also supports the crystalline model. Table I compares the maximal photoinduced birefringence, which is evaluated from Fig. 5, and the natural birefringence of the corresponding crystals. Here, following the above argument, we take the sign of the natural birefringence to be negative. We see that the photoinduced and the natural birefringence correlate in magnitude; Se exhibiting the greatest photoinduced birefringence, $\mathrm{As}_{2} \mathrm{~S}_{3}$ the next, and $\mathrm{As}_{2} \mathrm{Se}_{3}$ the smallest, the order being the same with that in the natural birefringence. The photoinduced birefringence is roughly $1 / 100$ of the crystalline value, the fraction which may reflect disordered amorphous structures. It seems difficult to explain this close correspondence between the photoinduced and the crystalline birefringence using other models which assume structural modifications in smaller atomic scales.

The temperature dependence shown in Fig. 4 is also consistent with the crystalline model. Here, we may assume, in a similar way to the conventional model describing crystal growths from melts, ${ }^{31}$ that illumination yields oriented crys- 
talline nuclei, which need thermal energy to grow. In the growth process, the glass-transition temperature may act like the melting temperature for the quasicrystals. Then, the photoinduced anisotropy can appear most efficiently at some temperature below the glass-transition temperature, which is consistent with the observations.

However, some problems remain unresolved. For instance, why the photoinduced birefringence is maximal for bandgap illumination at room temperature (Fig. 3) is not necessarily clear at present. The fact that $-\Delta n$ is smaller for light with $\hbar \omega<E_{g}$ may simply be due to the lack of excitation energy. Some excitations may occur, while these are overcome with isotropic thermal relaxation.

A problem is why $\Delta n$ decreases with an increase in $\hbar \omega$ at $\hbar \omega>E_{g}$. Here, the decrease can be related to the thermalization process of photoexcited carriers. ${ }^{32}$ It is known that photoexcited carriers thermally diffuse before recombination so that geminate recombination is suppressed. Quantitatively, the thermalization length in $\mathrm{Se}$ at room temperature ranges from $1 \mathrm{~nm}$ for $2.0 \mathrm{eV}$ excitation $\left(\hbar \omega \simeq E_{g}\right.$ ) to $5 \mathrm{~nm}$ for 2.8 $\mathrm{eV}$. For $\mathrm{As}_{2} \mathrm{~S}_{3}$ quantitative results have not been known, while a similar situation can be assumed. ${ }^{12}$ Then, if the thermalization extends too widely, recombination cannot give an energy efficiently to the site where the carriers are photoexcited. Alternatively, if the thermalization length is $\sim 1 \mathrm{~nm}$, geminate recombination may occur, which can give the energy efficiently to the excited atoms. Such a process may be responsible for growth of crystalline clusters with the aid of thermal energy. That is, energy localization seems to be needed for the structural change. The energy localization is responsible also for the photodarkening process, ${ }^{2,4}$ while it appears more prominently in the photoinduced anisotropy.

Following the above idea, we can conjecture a reason why $2.0 \mathrm{eV}$ light can provide a maximal birefringence at $400 \mathrm{~K}$ (Fig. 4). The fact can be ascribed to the temperature dependence of $E_{g}$, which decreases with increasing temperature. For instance, in $\mathrm{As}_{2} \mathrm{~S}_{3}, E_{g} \simeq 2.0 \mathrm{eV}$ at $400 \mathrm{~K},{ }^{3}$ and accordingly the idea described above may apply.

The crystalline model can provide a plausible explanation for the observations of the response times. It is known that the anisotropy after some illumination cycles increases more promptly than that induced in as-annealed glasses. ${ }^{19,20}$ In the present model, photoinduced crystalline clusters can be assumed to be more compact than original amorphous structures, since in general the crystalline state is more dense than the amorphous. If this is the case, the crystalline cluster may be able to rotate more easily, giving rise to a faster response.

Murayama has asserted that the crystalline model is inappropriate to understand the photoinduced anisotropy, with a reasoning that the polarized photoluminescence is observed in $\mathrm{As}_{2} \mathrm{~S}_{3}$ glass in contrast to the negative result in the crystal. ${ }^{12}$ However, in the present model, we can envisage heterogeneous structures in which photoinduced quasicrystalline clusters are embedded in amorphous networks. In such structures, the polarized photoluminescence would appear, since the quasicrystalline cluster could act as luminescence centers.

However, it is ambiguous at present whether the crystalline structure is natively contained in the amorphous structure and illumination is just responsible for the orientational change, or alternatively the original amorphous structure is completely random and illumination produces an anisotropic structures. This problem seems to be closely related with the nature of the FSDP, which is still a matter of controversy. ${ }^{33,34}$ If the so-called quasicrystalline structure model originally proposed for $\mathrm{As}_{2} \mathrm{~S}_{3}$ by Vaipolin and Porai-Koshits ${ }^{35}$ is pertinent, the layer-orientation model may be more plausible. However, if the original structure is a three-dimensional random network, the crystalline-growth model may be more appropriate.

It seems valuable to compare the present model with those proposed for the photoinduced anisotropy phenomena observed in other materials. We know at present that the photoinduced anisotropy is observed in many materials such as halide crystals including photographic materials, ${ }^{36,37}$ homogeneous and inhomogeneous oxide glasses, ${ }^{38-40}$ and organic materials. ${ }^{41,42}$ The mechanisms seem to consist of electronic and structural processes, the latter ranging between defect generation in relatively rigid materials $\mathrm{s}^{36,38}$ and molecular orientation in flexible organic materials. ${ }^{42}$ In these classifications, the present crystalline model can be understood as a kind of intermolecular-orientation models.

\section{CONCLUSIONS}

The mechanism of the photoinduced anisotropy widely observed in chalcogenide glasses has been studied through structural and optical investigations. These investigations suggest that the crystalline model is more appropriate to understand the overall features. The structural anisotropy seems to be induced most effectively by localized photoexcited carriers. Having obtained a model of the photoinduced anisotropy, the next study will be made upon the dynamics of the structural transformation.

\section{ACKNOWLEDGMENTS}

The authors would like to thank V. K. Tikhomirov, H. Hisakuni, and M. Notani for their experimental assistances. The present work was financially supported by grants from Ministry of Education, Suhara Memorial Foundation, Casio Science Foundation, Showa Electric Wire and Cable Co. Ltd., and Hitachi Microcomputer Engineering Ltd.
${ }^{1}$ Kazunobu Tanaka, in Fundamental Physics of Amorphous Semiconductors, edited by F. Yonezawa (Springer-Verlag, Berlin, 1981), p. 104.

${ }^{2}$ K. Tanaka, Rev. Solid State Sci. 4, 641 (1990).

${ }^{3} \mathrm{~S}$. R. Elliott, in Materials Science and Technology, edited by J. Zarzycki (VCH, Weinheim, 1991), Vol. 9, p. 375.
${ }^{4}$ K. Shimakawa, A. Kolobov, and S. R. Elliott, Adv. Phys. 44, 475 (1995).

${ }^{5}$ V. G. Zhdanov, B. T. Kolomiets, V. M. Lyubin, and V. K. Malinovsky, Phys. Status Solidi A 52, 621 (1979).

${ }^{6}$ V. G. Zhdanov, V. K. Malinovski, L. P. Nikolova, and T. Todorov, Opt. Commun. 30, 329 (1979). 
${ }^{7}$ J. Hajtó and P. J. S. Ewen, Phys. Status Solidi A 54, 385 (1979).

${ }^{8}$ J. Hajtó, I. Jánossy, and G. Forgács, J. Phys. C 15, 6293 (1981).

${ }^{9}$ R. Grigorovici, A. Vancu, and L. Ghita, J. Non-Cryst. Solids 59\&60, 909 (1983).

${ }^{10}$ K. Kimura, K. Murayama, and T. Ninomiya, J. Non-Cryst. Solids 77\&78, 1203 (1985).

${ }^{11}$ I. Jánossy, J. Hajto, and W. K. Choi, J. Non-Cryst. Solids 90, 529 (1987).

${ }^{12}$ K. Murayama, in Disordered Semiconductors, edited by M. A. Kastner, G. A. Tomas, and S. R. Ovshinsky (Plenum, New York, 1987), p. 185.

${ }^{13}$ J. M. Lee, M. A. Paesler, D. E. Sayers, and A. Fontaine, J. NonCryst. Solids 123, 295 (1990).

${ }^{14}$ T. Kosa and I. Jánossy, J. Non-Cryst. Solids 137\&138, 981 (1991).

${ }^{15}$ V. M. Lyubin and V. K. Tikhomirov, J. Non-Cryst. Solids $\mathbf{1 3 5}$, 37 (1991).

${ }^{16}$ H. Fritzsche, J. Non-Cryst. Solids 164-166, 1169 (1993); Phys. Rev. B 52, 15854 (1995).

${ }^{17}$ V. K. Tikhomirov and S. R. Elliott, Phys. Rev. B 49, 17476 (1994).

${ }^{18}$ V. K. Tikhomirov and S. R. Elliott, Phys. Rev. B 51, 5538 (1995)

${ }^{19}$ K. Tanaka, M. Notani, and H. Hisakuni, Solid State Commun. 95, 461 (1955).

${ }^{20}$ V. Lyubin and M. Klebanov, Phys. Rev. B 53, R11 924 (1996).

${ }^{21}$ C. H. Kwak, J. T. Kim, and S. S. Lee, Appl. Opt. 28, 737 (1989).

${ }^{22}$ H. M. Kim, J. W. Jeong, C. H. Kwak, and S. S. Lee, Appl. Opt. 34, 6008 (1995).

${ }^{23}$ K. Tanaka, J. Non-Cryst. Solids 119, 243 (1990); 119, 254 (1990).

${ }^{24}$ D. A. Holmes, J. Opt. Soc. Am. 54, 1115 (1964).

${ }^{25}$ K. Tanaka and H. Hisakuni, J. Non-Cryst. Solids 198-200, 714 (1996).

${ }^{26}$ The difference Fig. 1(b) appears to resemble Fig. 1(a), if the response at $\sim 19^{\circ}$ is neglected. This fact may suggest that the film illuminated with $x$-polarized light appears to be more ordered under inspections of the transmission arrangement. This idea is also consistent with the present model.

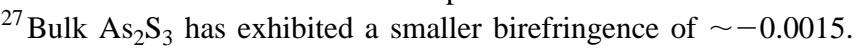

$\mathrm{As}_{35} \mathrm{~S}_{65}$ and $\mathrm{As}_{43} \mathrm{~S}_{57}$ glasses exhibited birefringences similar to and greater than that in $\mathrm{As}_{2} \mathrm{~S}_{3}$ glass, respectively.

${ }^{28}$ In some previous studies, annealing studies were made for nonstoichiometric glasses, which might cause different features. Actually, in bulk $\mathrm{As}_{2} \mathrm{~S}_{3}$, we have confirmed that $\Delta n$ induced at room temperature is stable up to $400 \mathrm{~K}$. It should also be noted that in some previous studies the optical anisotropy may not be evaluated accurately, since light intensity transmitted through a sample is monitored, which can be affected by birefringence, dichroism and changes in the refractive index and the absorption coefficient [see, H. Hisakuni and K. Tanaka, Solid State Commun. 90, 483 (1994)].

${ }^{29}$ R. Zallen and D. F. Blossey, in Physics and Chemistry of Materials with Layered Structures, edited by E. Mooser (Reidel, Dordrecht, 1976), p. 231.

${ }^{30}$ In the crystalline $\mathrm{As}_{2} \mathrm{~S}_{3}$ the layer plane is defined by the $a$ and $c$ axes. The in-plane refractive index is taken to be the average for these directions.

${ }^{31}$ B. Lewis, in Crystal Growth, edited by B. Pamplin (Pergamon, Oxford, 1980), p. 23.

${ }^{32}$ J. Mort and J. Knight, Nature (London) 290, 659 (1981); J. Mort, J. Phys. C 4, 433 (1981).

${ }^{33}$ S. R. Elliott, Nature (London) 354, 445 (1991).

${ }^{34}$ I. T. Penfold and P. S. Salmon, Phys. Rev. Lett. 67, 97 (1991).

${ }^{35}$ A. A. Vaipolin and E. A. Porai-Koshits, Fiz. Tverd. Tela (Leningrad) 5, 683 (1963) [Sov. Phys.-Solid State 5, 497 (1963)].

${ }^{36}$ F. Caimi, Opt. Eng. 17, 327 (1978).

${ }^{37}$ N. F. Borrelli, J. B. Chodak, and G. B. Hares, J. Appl. Phys. 50, 5978 (1979).

${ }^{38}$ J. H. Stathis, Phys. Rev. Lett. 58, 1448 (1987).

${ }^{39}$ A. L. Huston and B. L. Justus, Opt. Lett. 20, 952 (1995).

${ }^{40}$ D. C. Psaila, F. Quellette, and C. M. de Sterke, Appl. Phys. Lett. 68, 900 (1995).

${ }^{41}$ T. D. Edralidze and N. A. Edralidze, Appl. Opt. 31, 4720 (1992).

${ }^{42}$ M. Schadt, H. Seiberle, A. Schuster, and S. M. Kelly, Jpn. J. Appl. Phys. 34, L764 (1995).

${ }^{43}$ W. C. Cooper, K. G. Bennett, and F. C. Croxton, in Selenium, edited by R. A. Zingaro and W. C. Cooper (Van Nostrand Reinhold, New York, 1974), p. 1.

${ }^{44}$ E. Tarnow, A. Antonelli, and J. D. Joannopoulos, Phys. Rev. B 34, 8718 (1986). 